\title{
Agrocombustibles y cambio climático en la Orinoquia colombiana
}

\author{
Biofuels and climate change in \\ the colombian Orinoco river basin
}

\section{Agrocombustíveis e Mudanças Climáticas na Orinoquia Colombiana}

\author{
Óscar Emerson Zúnigiga Mosquera \\ Engenheiro Agrônomo - Universidad Nacional de Colombia, \\ Magister em Desenvolvimento e Meio Ambiente - PRODEMA / UFPE \\ Pesquisador no Grupo de Pesquisas em Desenvolvimento e Meio Ambiente - IFPE
}

oscar.emersonzuniga@ufpe.br

\section{Resumen}

En las últimas décadas la búsqueda de recursos naturales reconfiguró el mapa geopolítico mundial, a través de nuevas alianzas de carácter político-económico. Estudios regionales colocan al subcontinente suramericano como un escenario de posible disputa comercial entre dos polos: el emergente BRICS y los viejos países industrializados. La región colombiana conocida como la Altiplanicie llanera es el epicentro de nuevas inversiones económicas por parte de capitales nacionales e internacionales con la intención de desarrollar proyectos de tipo agronegocio, entre los que se puede encontrar la producción de biocombustibles sustentados en el discurso del cambio climático. Este artículo presenta resultados del trabajo de investigación sobre el tema de Índice de Vulnerabilidad Territorial, visibilizando los nudos que se encuentran y legitiman las transformaciones materiales junto con producciones discursivas que moldean y diseñan el territorio. Parte de este trabajo fue presentando en el / Seminario Internacional Novas territorialidades e desenvolvimento sustentavél, realizado en Brasil en 2011.

Palabras clave: geopolítica de los recursos naturales, inversión económica, vulnerabilidad territorial, territorio.

\section{Abstract}

In recent decades, the search for natural resources has reconfigured the geopolitical map through new political-economic alliances. Regional studies identify the South American continent as the scene for a potential trade dispute between two poles: the emerging BRICS and the old industrialized countries. The Colombian region known as the highland plains is the epicenter of new economic investments by domestic and international stakeholders who have the intention of developing agribusiness projects, among which promote the biofuels production at the center of the climate change debate. This paper presents research results on the topic of the Territorial Vulnerability Index, bringing to light some of the knots encountered and that legitimize material transformations alongside discursive productions that shape and design the territory. Part of this paper was presented at the I Seminario Internacional Novas Territorialidades e Desenvolvimento Sustentavél, held in Brazil in 2011.

Key-words: natural resource geopolitics, economic investment, territorial vulnerability, territory

\section{Resumo}

Nas últimas décadas a busca por recursos naturais reconfigurou o mapa geopolítico global através 
de novas alianças de caráter político-econômico. Estudos regionais colocam o subcontinente sul-americano como um cenário de possível disputa comercial entre dois pólos: o emergente BRICS e os antigos países industrializados. A região da Colômbia conhecida como altiplanicie llanera é o epicentro de novos investimentos econômicos de capital nacional e internacional, com a intenção de desenvolver projetos do tipo agronegócio, entre os quais se encontra a produção de biocombustíveis apoiado no discurso da mudança climática. Este trabalho apresenta resultados de pesquisa sobre o tema do Índice de Vulnerabilidade Territorial, visibilizando os pontos que se encontram e legitimam as transformações materiais em parceria com as produções que moldam o território. Parte deste trabalho foi apresentada no I Seminário Internacional Novas Territorialidades e Desenvolvimento Sustentável, realizada no Brasil em 2011.

Palavras-chave: geopolítica dos recursos naturais, investimento econômico, vulnerabilidade territorial, território.

\section{Introducción}

\section{El territorio como categoría de estudio}

El marco de análisis considerado en este trabajo es la producción y sus relaciones. Llevando el hilo conductor de la producción, considerase la categoría de estudio territorio para identificar los vínculos económicos, nacionales e internacionales, que permiten la producción y reproducción del capital, generando así espacios luminosos y espacios opacos (Santos; Silveira, 2008, p. 264).

Teniendo en cuenta el territorio como espacio humano creado y transformado por y para la producción, el agronegocio y los agrocombustibles se convierten en tema clave para comprender los cambios que actualmente presenta la Orinoquía colombiana.

\section{Caracterización de los agrocombustibles}

Los agrocombustibles se presentan en dos formas: el etanol y el biodiesel. El primero es un alcohol menos contaminante, en emisiones de $\mathrm{CO}_{2}$, por no tener mezcla con derivados de fuentes fósiles, siendo la caña de azúcar la materia prima más usada.

El biodiesel, por el contrario, es una mezcla de aceites de origen vegetal o grasa animal con formas de energía fósil. Según el tipo de mezclas que se realicen, modificando la concentración de las fuentes aceites vegetales o animales, el biodiesel se clasifica desde B2 (menos puro) hasta B100 (sin fuente de energía fosilizada). ${ }^{1}$

Esta capacidad de reducir las emisiones de $\mathrm{CO}_{2}$ y tener un mayor poder calorífico ha colocado al biodiesel como un producto clave en la agenda de mitigación del calentamiento global y el cambio climático, que gracias al protocolo de Kioto, los valoriza como mercancía dentro de la economía verde.

Sin embargo, más allá del discurso del cambio climático, los agrocombustibles traen los mismos problemas que otros proyectos de tipo agronegocio tales como: precarias condiciones laborales, concentración de tierra, establecimiento de monocultivos y actividad empresarial excluyente pues la agricultura familiar no tiene espacio rentable dentro de este tipo de inversiones. Según Wilkinson, la estructura del gran capital global, que posee inversiones en la producción o comercialización de los agrocombustibles, está dividida en dos formas: el capital que busca inversiones rentables, como por ejemplo los fondos de inversiones y las empresas que ya están en el sector y buscan monopolizar toda la cadena productiva (Wilkinson et. al., 2008).

1 Cfr. www.minagricultura.gov.co/02componentes/05biocom bustible.aspx\# 


\section{Características de la región}

La república de Colombia está localizada en el noroeste de América de Sur. Al norte limita con la República de Panamá y el Mar Caribe; al este con la República Bolivariana de Venezuela; al sur con Perú, Ecuador y Brasil. Posee 1.141.748km² de área terrestre, que sumados a la plataforma continental dan $2.070 .408 \mathrm{~km}$. Administrativamente se divide en 32 departamentos y un Distrito Capital llamado Bogotá. En la zona sudeste de Colombia se encuentra la Orinoquia².

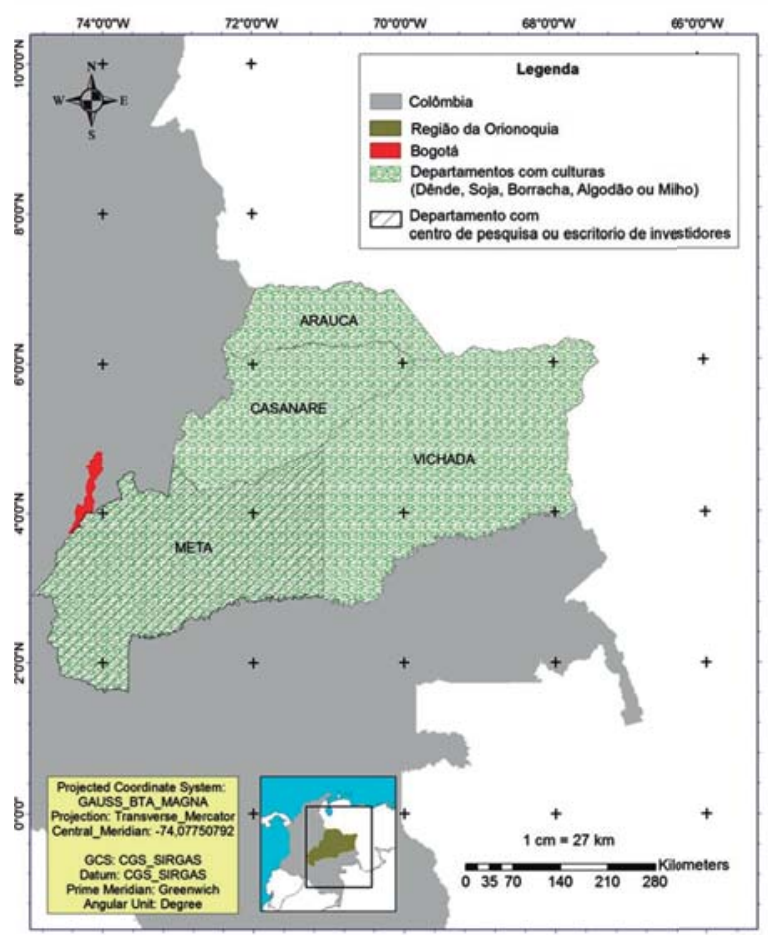

Figura 1. Localización de la Orinoquia en la Republica de Colombia.

Fuente: Autor.

La Orinoquia es un importante centro agropecuario del país, especialmente en la producción de ganado. A partir de finales del siglo XX, se convirtió en una región económicamente activa debido a la implementación de cultivos como la caña de azúcar, palma de aceite, caucho, arroz, maíz y soya.

2 Colombia está dividida en seis regiones biogeográficas: Amazonia, Andina, Caribe, Insular, Orinoquía y Pacífica

\section{La Internacionalización de la Orinoquia a través de los agrocombustibles}

En Colombia el principal cultivo para la obtención de biodiesel es la palma de aceite (Elaeis guineensis Jacq). Los departamentos con mayor número de cultivos, en orden de importancia, son: Meta, Cesar, Santander, Magdalena, Nariño, Casanare, Bolívar, Cundinamarca y Norte de Santander (Figura 2). Además, en municipios de la región se han establecidos como prioridad otros cultivos para la producción de combustibles, como parte de una agenda de productividad, entre estos están: soja, caña y yuca. (República de Colombia, 2009).

Un dato que muestra la importancia de la región en el contexto internacional es el índice de competitividad, realizado por la Comisión Económica para América Latina y el Caribe - CEPAL. En tanto otros índices están por debajo de la media ${ }^{3}$, el índice de internacionalización de la economía consiguió el mayor valor entre todos, 23/23. (República de Colombia, 2009).

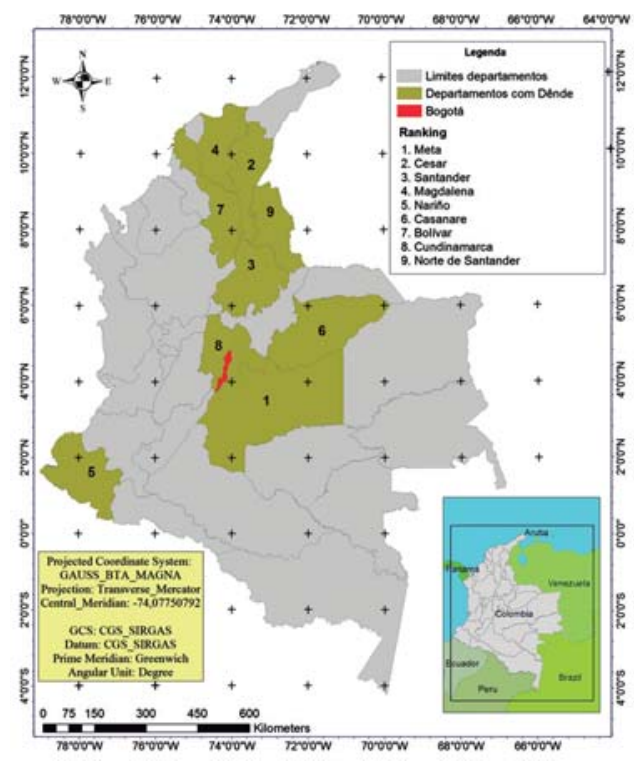

Figura 2. Ranking de los departamentos con cultivos de palma de aceite para la producción de biodiesel.

Fuente: Adaptado de Fedepalma 2011.

${ }^{*}$ ) La numeración representa la jerarquía en el cultivo de palma, así el departamento del Meta ocupa el primer lugar en la producción de palma y el departamento de Norte de Santander el último del ranking.

3 Como es el caso de: Poder Económico evaluado (6/23); Gobierno e instituciones (16/23); Finanzas (12/23) y Recursos Humanos (12/23). 
Actualmente la región es el centro del debate de la ley 160 de 1994, la cual limita la capacidad de compra de tierra en la región a una Unidad Agrícola Familiar (UAF) 4 . Sin embargo, los empresarios extranjeros han realizado maniobras para conseguir comprar grandes cantidades de tierra para el establecimiento del agronegocio.

Entre los principales compradores de tierra de la región están firmas chinas y brasileras. La figura 3 muestra la relación entre inversiones nacionales y extranjeras, el establecimiento de centros de investigación y cultivos.

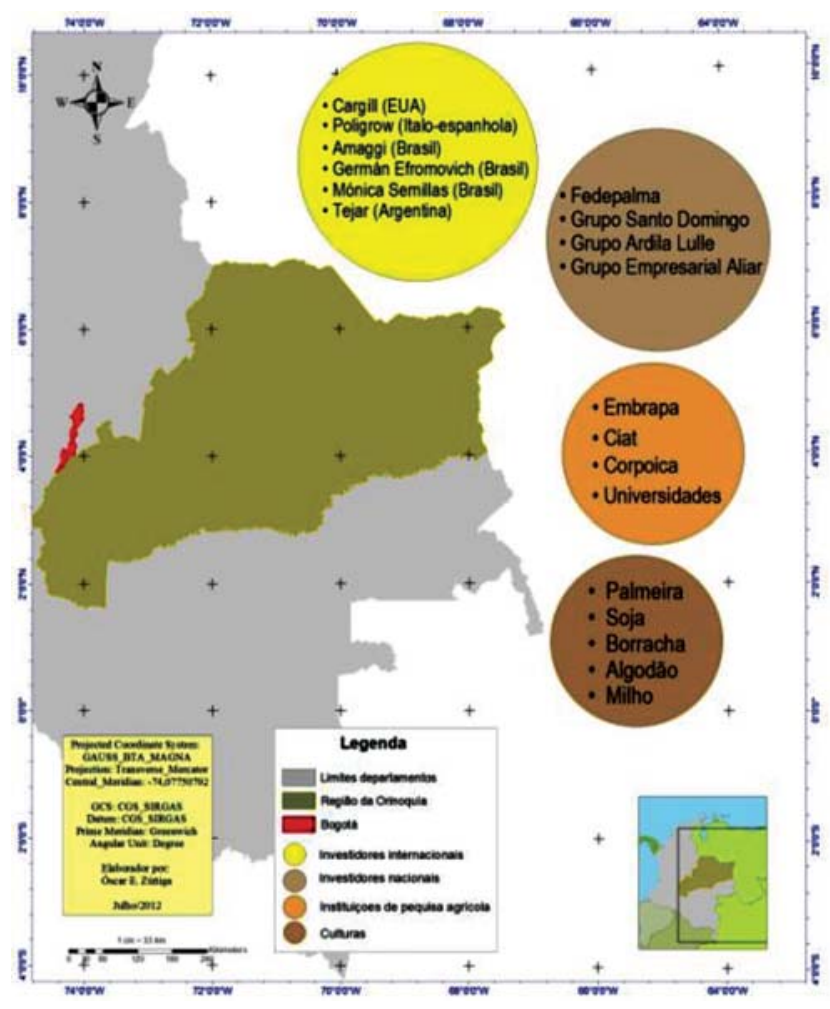

Figura 3. Inversiones presentes en la Orinoquia en la República de Colombia.

Fuente: Revista Dinero, 2011. Organizado por: el autor

4 La ley colombiana define a UAF como el núcleo de producción agrícola, pecuaria, acuicultura y de extensión forestal que permite a la familia trabajar y formar su patrimonio $A$ lei define a UAF como a empresa do núcleo de produção agrícola, pecuária, aquicultura e extensão florestal que permite a família trabalhar e a formação de seu patrimônio.
El mercado de los agrocombustibles en Colombia ha sido fuertemente estimulado por el discurso del cambio climático. Un ejemplo de ello es la ley № 629 , de 27 de diciembre de 2000, donde el país ratifica el Protocolo de Kioto y establece junto a los países del Anexo I (Naciones Unidas, 1992, 1998) objetivos de desarrollar auxilios para la producción y uso de agrocombustibles y reducción de gases efecto invernadero (IPCC, 2001).

Dentro de los sectores beneficiados con este acuerdo se encuentra el palmicultor. Dicho sector anunció en el 2009 que la Organización de las Naciones Unidas (ONU) aprobó el Proyecto Sectorial Sombrilla MDL para la captura de metano, desplazamiento de combustibles fósiles y cogeneración de energía renovable. De acuerdo con el estudio de pre-factibilidad de potencial de creación de créditos de reducción de emisiones, para el sector están "dadas las condiciones del mercado internacional de carbono, los ingresos adicionales por año por MDL podrán ser entre US $\$ 1$ ' 885,000 y US $\$ 2 ' 639,000$. Estos ingresos se generarían por 10, 14 o 21 años, y el precio podría variar hacia arriba, mantenerse estable, o reducirse, dependiendo en el rumbo eventual del mercado y la estrategia de colocación de los CER" (Centro Andino para la Economía en el Medio Ambiente, 2004. p.15).

\section{Consideraciones finales}

El avance de las inversiones de agrocombustibles, especialmente para biodiesel, se sustenta en por lo menos tres factores, que sirven como metodología de estudio para las transformaciones territoriales: un discurso legitimador, la transformacional física del espacio y un marco de producción.

El discurso legitimador es el del cambio climático cuya hipótesis del calentamiento global por incremento de gases de invernadero y en especial el $\mathrm{CO}_{2}$, aún se encuentran en debate por la comunidad científica. 
Las transformaciones físicas del espacio están vinculadas al avance técnico-científico-informacional bajo los intereses empresariales que crean espacios de alto desarrollo ingenieril e modernización (luminosos) y otros que conviven con los anteriores definidos como opacos.

El marco de producción, que al final es la justificativa de las dos anteriores, se vincula con el surgimiento de las preocupaciones ambientales y de sustentabilidad, expresadas en términos de mitigación de emisiones de $\mathrm{CO}_{2}$.

\section{Literatura citada}

1. Cardoso, E. \& Holland, M. (2010). ¿Sudamérica para los chinos?: Un análisis basado en el comercio. Madrid: Fundación Carolina, 70p. Disponíble: http://www.fundacioncarolina.es. Acceso el: 09 de mayo de 2011.

2. Centro Andino para la Economía en el Medio Ambiente. (2004). Estudio sobre prefactibilidad para la identificacion y evaluacion del potencial de generacion de certificados de reduccion de emisiones a traves delmecanismo de desarrollo limpio para el sector palmero en colombia.
Resumen ejecutivo. Agosto. Disponíble en: www.fedepalma.org/documen/2005/resumen ejecutivo MDL. doc. Acceso el 09 de mayo de 2011.

3. Fedepalma. (2010). La palma de aceite. Disponívble en: <http://www.fedepalma.org/palma.htm> Acceso ee 22 de Nov de 2010.

4. Grupo Intergubernamental de Expertos Sobre Cambio Climático. (2001). Tercer Informe de Evaluación. Cambio Climático. Impactos, adaptacion y vulnerabilidad. Suiza: IPCC. 92 p.

5. Naciones Unidas. (1992). Convenio Marco de las Naciones Unidas sobre Cambio Climático.

6. Naciones Unidas. (1998). Protocolo de Kyoto de la convención marco de las naciones unidas sobre el cambio climático, Naciones Unidas.

7. República de Colombia. (2009). Plan prospectivo agropecuario Puerto Gaitán 2020. Puerto Gaitán. 361 p.

8. Revista Dinero. Los nuevos Ilaneros. Disponíble en: http:// www.dinero.com/caratula/edicion-impresa/articulo/losnuevos-llaneros/113492 Acceso el: 26 de febrero 2011.

9. Santos, M. \& Silveira, M. L. (2008). O Brasil: território e sociedade no inicio do século XXI. 12 ed. Rio de Janeiro, Editora Record, $471 \mathrm{p}$.

10. Wilkinson, J. \& Herrera, S. (2008). Subsidios para a discussão dos agrocombustivies no Brasil. In: Maia, K. et. al. (org.) Agrocombustiveís e a agricultura familiar e camponesa: subsídios ao debate. Rio de janeiro: REBRIP/FASE. Disponíble en:<www.boell-latinoamerica. org> Acceso el: 13, Jan, 2011. p. 24-57. 